\title{
Preparing Disease Surveillance Systems for ICD10
}

\author{
Alimelu Jonnagadla*2, Wayne Loschen ${ }^{2}$, Julia Gunn ${ }^{1}$ and Jennifer Evans ${ }^{1}$ \\ ${ }^{1}$ Boston Public Health Commission, Boston, MA, USA; ${ }^{2}$ Johns Hopkins University Applied Physics Laboratory, Laurel, MD, USA
}

\section{Objective}

To help users seamlessly query and analyze data in disease surveillance systems using both ICD9 and ICD10 codes during the transition period. Additionally, the mappings between ICD9 and ICD10 codes must be flexible enough to support locally required changes based upon a user's needs.

\section{Introduction}

The compliance date for the ICD9-ICD10 transition is October 1, 2015. The hospitals have started the ICD9-ICD10 transition. However, not all data providers will transition the data at the same time. In order to facilitate some coherence to the data during this transition period, user interface and data processing functionalities have been developed in ESSENCE to allow usage of both classification systems simultaneously. This capability will allow users to perform ICD10based queries across all the hospitals in their system, irrespective of the exact number of hospitals that have completed the ICD10 transition.

\section{Methods}

The Boston Public Health Commission utilizes an ESSENCE system to perform disease surveillance in their community. This project enhanced their existing ESSENCE system to support ICD10based queries. Using the Centers for Medicare \& Medicaid Services (CMS) and the Centers for Disease Control and Prevention's (CDC) General Equivalence Mappings (GEMs) as a starting point, processes were built to convert the ICD9 codes in both existing and incoming data to the equivalent ICD10 codes. This newly derived information is stored in a new field to allow the system to query against it, while keeping the original information for users to view as well. The user interface to perform queries in ESSENCE was updated to support ICD10-based filtering. Additionally, the interface provides an ICD10 lookup mechanism using codes and keywords to help users learn or remember the ICD10 codes they may need for a query. Also, a user interface and a backend process were built to provide administrators the ability to modify the ICD9-ICD10 mappings as needed. This gives the administrators the mechanism to adjust the mappings based upon their needs or to respond to coding patterns of their specific hospitals. Finally, the system provides a mechanism to reprocess the entire historical dataset using the modified mappings when required.

\section{Results}

The above described ESSENCE functionality has been implemented and deployed to the Boston Public Health Commission system. The described features have enabled the public health professionals to query the data using ICD10 codes without any concern of which code classification the data source originally included. This new capability also provides users with the ability to perform yearly reporting even during a transition year. This has allowed a hospital that has 9 months of ICD-9-CM data and three months of ICD-10-CM/PCS data in same year to still be queried on and provide usable results.

\section{Conclusions}

Public health entities will face many challenges due to the transition of the ICD code set. Adding the ability to convert to ICD10 codes and provide a single query function to ESSENCE well in advance of the compliance date will ease the transition and allow users to still utilize the hospital's data during the change. Providing the flexibility for a system to modify the mappings will allow for local behaviors in coding practices to be accounted for that otherwise may be lost by using a single mapping system.

\section{Keywords}

ICD10; Disease Surveillance; ESSENCE; ICD9-ICD10 transition

\section{Acknowledgments}

This work was funded by Boston Public Health Commission

*Alimelu Jonnagadla

E-mail: jonnaas1@jhuapl.edu 